\title{
EEG Source Reconstruction using Sparse Basis Function Representations
}

\author{
Hansen, Sofie Therese; Hansen, Lars Kai
}

Published in:

Proceedings of 4th International Workshop on Pattern Recognition in Neuroimaging

Publication date:

2014

Document Version

Peer reviewed version

Link back to DTU Orbit

Citation (APA):

Hansen, S. T., \& Hansen, L. K. (2014). EEG Source Reconstruction using Sparse Basis Function

Representations. In Proceedings of 4th International Workshop on Pattern Recognition in Neuroimaging IEEE.

\section{General rights}

Copyright and moral rights for the publications made accessible in the public portal are retained by the authors and/or other copyright owners and it is a condition of accessing publications that users recognise and abide by the legal requirements associated with these rights.

- Users may download and print one copy of any publication from the public portal for the purpose of private study or research.

- You may not further distribute the material or use it for any profit-making activity or commercial gain

- You may freely distribute the URL identifying the publication in the public portal

If you believe that this document breaches copyright please contact us providing details, and we will remove access to the work immediately and investigate your claim. 


\title{
EEG Source Reconstruction using Sparse Basis Function Representations
}

\author{
Sofie Therese Hansen and Lars Kai Hansen \\ Technical University of Denmark \\ Department of Applied Mathematics and Computer Science \\ Kgs. Lyngby, Denmark, (sofha,lkai@dtu.dk)
}

\begin{abstract}
State of the art performance of 3D EEG imaging is based on reconstruction using spatial basis function representations. In this work we augment the Variational Garrote (VG) approach for sparse approximation to incorporate spatial basis functions. As VG handles the bias variance trade-off with cross-validation this approach is more automated than competing approaches such as Multiple Sparse Priors (Friston et al., 2008) or Champagne (Wipf et al., 2010) that require manual selection of noise level and auxiliary signal free data, respectively. Finally, we propose an unbiased estimator of the reproducibility of the reconstructed activation time course based on a split-half resampling protocol.
\end{abstract}

\section{INTRODUCTION}

We are interested in fast and accurate reconstruction of the sources of macroscopic EEG signals measured by surface electrodes. The aim of the reconstruction is to infer the spatio-temporal structure of the source signals supporting our long term goal: To combine EEG and behavioral data with neuroscience databases for real-time annotated and interpreted brain monitoring. The lack of spatial precision is one of the major challenges to such a program. The state of the art in high accuracy spatio-temporal reconstruction is represented by the multiple sparse priors (MSP) approach [1], [2], based on spatial basis functions and an approximate inference scheme using socalled automatic relevance determination (ARD) [3], [4], [5], and the Champagne framework [6], [7]. These methods are based on a combination of approximate Bayesian inference and simple heuristics, such as tuning temporal complexity by the Kaiser criterion and weakly informative hyper priors [1] or use noise estimates from other data [6]. The implicit regularization in ARD, leading to sparse solutions, is implemented by the divergence of certain precision parameters forming a highly complex optimization problem [8], [9]. Here we explore an alternative approach which combines approximate Bayesian inference and resampling methods for a more automated approach to source reconstruction in relevant spatial bases without sacrificing accuracy or speed. The main contributions in this work are the following 1) We show that the MSP basis function approach can easily be adapted to the Variational Garrote (VG). 2) In the VG an unbiased cross-validation step is used to optimize and validate sparsity. 3) We introduce an unbiased measure of the reproducibility of the activation time course based on a split-half resampling protocol.

\section{THE EEG INVERSE PROBLEM}

Reconstruction of the EEG source distribution is based on the well-established linear relation between the measured scalp EEG potentials and the cortex level EEG generators [10], [11], [12], [13], [2]. Noting that the inverse mapping from electrodes to cortical sources is highly ill-posed, regularization is needed. We follow a Bayesian approach and control complexity by assigning priors. Sparsity of the solution is a well-known means for complexity control and at the same time motivated for EEG by recent work on the dipolar nature of independent signal components [14]. The Variational Garrote (VG), originally proposed by Kappen et al. [15] has been adapted to the EEG reconstruction problem [16]. The approach promotes sparse solutions by introducing a binary variable for each potential dipole location, implementing a flexible and adaptive description of the dipole support. A spatio-temporal multiple measurements vector approach is obtained by the additional assumption that the support is constant within a given time window (time expanded VG, teVG) [17].

The simple VG/teVG modifications to the linear regression problem, relating the EEG potentials $\mathbf{Y} \in \mathbb{R}^{K \times T}$ to the sources $\mathbf{X} \in \mathbb{R}^{N \times T}$ through lead fields $\mathbf{A} \in \mathbb{R}^{K \times N}$, are

$$
\begin{array}{rlrl} 
& \text { Linear reg. } & Y_{k t} & =\sum_{n=1}^{N} A_{k n} X_{n t}+\text { noise } \\
\text { VG } & Y_{k t} & =\sum_{n=1}^{N} A_{k n} S_{n t} X_{n t}+\text { noise } \\
\text { teVG } & Y_{k t} & =\sum_{n=1}^{N} A_{k n} s_{n} X_{n t}+\text { noise }
\end{array}
$$

where $S_{n t}$ and $s_{n}$ are binary variables $\in\{0,1\}$ and the additive noise is assumed to be Gaussian with zero mean. Using variational approximation, as described by [15] the solutions to VG and teVG can be found. Note that computation time is markedly reduced in teVG compared to individual VG reconstructions for each time point [17]. Furthermore source recovery is also greatly improved [17]. In the following, teVG is augmented to incorporate spatial basis functions. In order to make teVG more comparable with MSP, we assume in the following constant support across the whole time frame. It could be argued that dividing the data into smaller time windows would be more appropriate as brain activity can happen on short time scales [18].

\section{A. Implementation of Basis Functions}

Basis functions are included to model the synchronous activity seen between neighboring neurons [20]. Following the framework of constructing spatial source components in MSP [1], we sample the basis functions from a coherence matrix based on the Green's function encompassing the connectivity of the cortical mesh [1]. The outcome is a set of source basis 


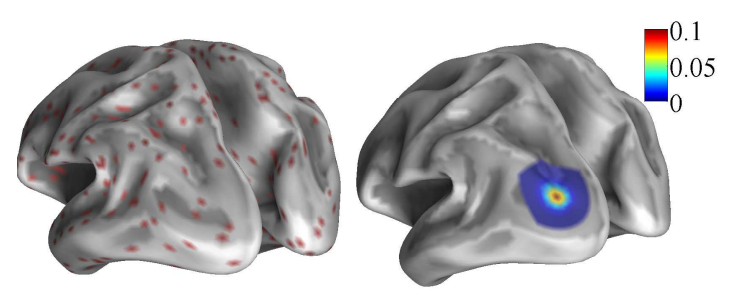

Fig. 1. Projection of the basis functions to the cortex. Left: center vertices (in red) of the $3 \times 256$ source components, and right: the spatial extent of one component. Note that the 256 bilateral components completely overlap with the 256 left and 256 right hemisphere components.

functions with compact support, see example in Fig. 1, which also shows the center vertices of 768 components.

We sample $C / 3$ basis functions per hemisphere and additionally create $C / 3$ bilateral functions from the unilateral functions. The $C$ basis functions can therefore model both unilateral and bilateral activity. The set of functions, make up the basis $\mathbf{B} \in \mathbb{R}^{N \times C}$. Transforming the regression problem, it now relates the observations across time $\mathbf{Y} \in \mathbb{R}^{K \times T}$ to the source functions $\mathbf{X}_{C} \in \mathbb{R}^{C \times T}$ through the lead fields $\mathbf{A} \in \mathbb{R}^{K \times N}$ and the basis $\mathbf{B}$

$$
\mathbf{Y}=\mathbf{A B X}_{C}+\text { noise } \Longleftrightarrow \mathbf{Y}=\mathbf{D X}_{C}+\text { noise },
$$

where $\mathbf{D} \in \mathbb{R}^{K \times C}$. Following equations (1)-(3) teVG is easily applied to the constructed basis functions, where we now have $C$ binary variables. Note, the source functions are projected back to the original source space by $\mathbf{X}=\mathbf{B X}_{C}$. In the following analyses $C=768$ basis functions are applied.

\section{Simulations}

Through simulations emulating real EEG the localization accuracy is investigated. Two areas of activation are simulated having temporal dynamics as shown in Fig. 2, upper panel. Their locations correspond to the left and right fusiform face areas (FFAs) as found by Henson et al. (2003) [19] in an fMRI study. The peak activity in the left and right FFAs were reported in MNI space at $(-39,-51,-24) \mathrm{mm}$ and $(42,-45,-27) \mathrm{mm}$, respectively. Each FFA is simulated to cover seven sources whose locations can be seen in Fig. 3(a). The forward field matrix employed is described in the next section where actual EEG source reconstruction is performed [21], [22]. The dimensions of the current simulation therefore correspond to the upcoming EEG analysis, where the cortex is divided into $N=8196$ vertices and the EEG is measured through $K=128$ electrodes.

The teVG is compared to MSP across 1000 simulations at signal to noise ratios (SNRs), 1 and $10^{1}$. Both methods apply

\footnotetext{
${ }^{1} \mathrm{SNR}=\langle\operatorname{Var}($ Signal $) / \operatorname{Var}($ Noise $)\rangle$ where $\langle\ldots\rangle$ is the time average.
}

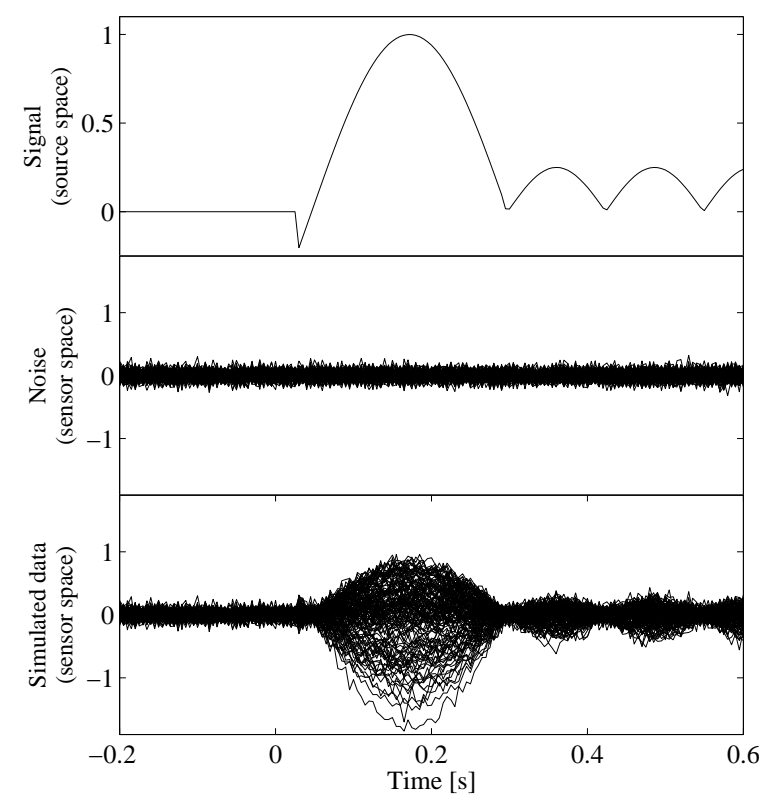

Fig. 2. Simulated data at source level together with noise and simulated data at sensor level. Seven sources having activity equal to top panel are planted in the left and right FFA. The location of these two patches can be seen in Fig. 3(a). Signal is projected to sensor space using a forward model and white noise (middle panel) is added to give the signal in the bottom panel, here $\mathrm{SNR}=10$.

the described spatial basis functions. The temporal complexity is controlled in MSP by dimensionality reduction using principal component analysis, with a heuristic variance threshold applied to the time series before applying the ARD inference scheme. The sparsity level in teVG is selected in four-fold cross-validation (with resampling of electrodes), while the noise variance is estimated by the Bayesian scheme.

The mean localization errors are reported in Table I and the mean solutions for SNR $=10$ are visualized in figures 3(b) and (c) using MSP and teVG respectively. The overall impression from Fig. 3 is that the teVG reconstruction is more focal, while MSP produces more scattered activity by reconstructing dipoles further away from the true activity, i.e. in the mid frontal/parietal and anterior temporal regions. Comparing in Table I MSP and teVG we find that MSP is on average $0.3 \mathrm{~mm}$ more accurate, hence, the more automatic teVG approach leads to localization results that are close to state of the art methods. For both methods the time complexity of reconstruction depends on a number of choices (number of iterations etc.), here we have tuned these parameters to result in approximately the same time complexity (a total of $\Delta=10$ $15 \mathrm{~s}$ including cross-validation in teVG).

In a related experiment the FFA patches reported in [19] are moved to the location of their respective nearest source

TABLE I. LOCALIZATION ERRORS FOR TEVG AND MSP ON SIMULATED LEFT (L) AND RIGHT (R) FFA ACTIVITY. THE ERROR IS MEASURED AS THE EUCLIDEAN DISTANCE BETWEEN THE CENTER OF THE PLANTED ACTIVITY (IN THE LEFT OR RIGHT HEMISPHERE) TO THE SOURCE ESTIMATED TO HAVE LARGEST MAGNITUDE (IN THE LEFT OR RIGHT HEMISPHERE). THE MEANS OF 1000 REPETITIONS ARE LISTED. DUE TO THE HIGH NUMBER OF REPEATS, STANDARD DEVIATIONS OF THE MEANS ARE ALL BELOW 0.4 MM RENDERING ALL POSITIVE AND NEGATIVE DIFFERENCES BETWEEN MSP AND TEVG LOCALIZATION SIGNIFICANT $(p<0.05)$. THE OVERALL MEAN LOCALIZATION ERRORS ARE VERY SIMILAR (MSP $=18.3 \mathrm{MM} \mathrm{AND} \mathrm{TEVG=18.6} \mathrm{MM).}$

\begin{tabular}{c|c|c|c|c}
\hline & \multicolumn{2}{|c|}{ FFAs from Henson et al. (2003) [19] } & \multicolumn{2}{c}{ FFAs from Henson et al. (2003) [19] projected to nearest basis function center } \\
\hline & SNR $=1$ & SNR $=10$ & SNR $=1$ & SNR $=10$ \\
\hline MSP & L: $13.8 \mathrm{~mm} ;$ R: $14.5 \mathrm{~mm}$ & L:10.8 $\mathrm{mm} ; \mathrm{R}: 31.9 \mathrm{~mm}$ & L:13.4; R: $18.7 \mathrm{~mm}$ & L: $15.1 \mathrm{~mm} ; \mathrm{R}: 27.9 \mathrm{~mm}$ \\
\hline teVG & L: $19.1 \mathrm{~mm} ; \mathrm{R}: 22.8 \mathrm{~mm}$ & L: $18.0 \mathrm{~mm} ; \mathrm{R}: 21.6 \mathrm{~mm}$ & L: $17.7 \mathrm{~mm} ; \mathrm{R}: 21.1 \mathrm{~mm}$ & L: $16.3 \mathrm{~mm} ;$ R: $12.2 \mathrm{~mm}$ \\
\hline
\end{tabular}



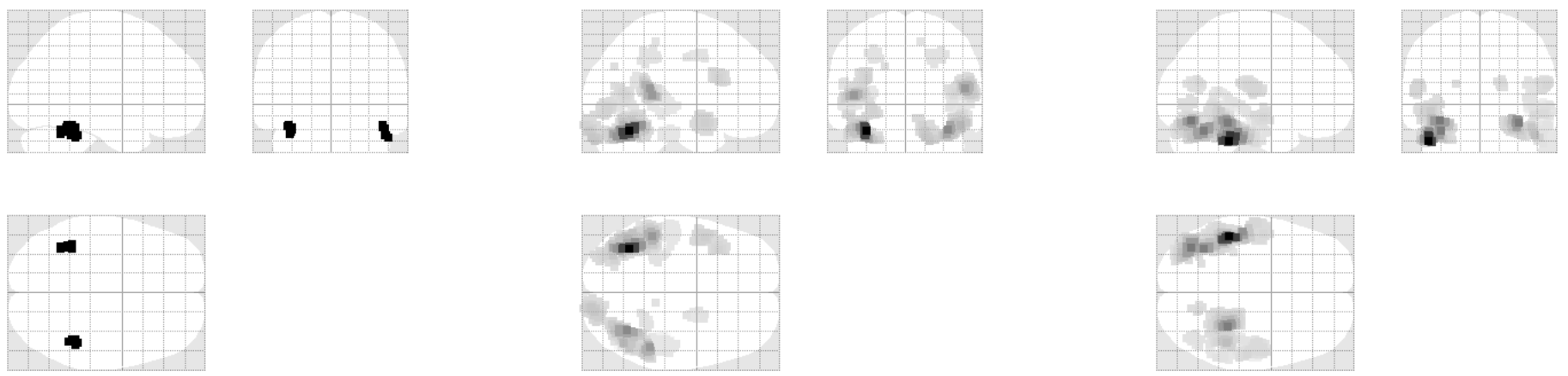

(a) True

(b) MSP

(c) teVG

Fig. 3. Glass brain view of the simulated FFA source distribution, and the estimates by MSP and teVG, respectively. The two latter are averages across 1000 repetitions of reconstructions of the true response added with noise yielding an $\mathrm{SNR}=10$. Shown are 512 dipoles.

function centers. The aim here is to investigate whether the localization error decreases when the actual sources are close to a basis function center. The results are seen in Table I. The relative insignificant changes indicate that selection of basis functions is not critical to the result.

\section{EEG ANALYSIS}

We now test teVG with basis functions on a benchmark 128-channel EEG data set from a subject viewing faces and scrambled faces. The paradigm of the face perception study is described in [19] and the data can be accessed via http:// www.fil.ion.ucl.ac.uk/spm/data/mmfaces/. The forward model used here, is a symmetric BEM head model produced in SPM8 (http://www.fil.ion.ucl.ac.uk/spm/) with the open source plugin OpenMEEG [22]. As earlier, the cortex is divided into $N=8196$ vertices which are projected to $C=768$ basis functions. About 150 epochs are recorded of each of the two conditions. The source reconstructed difference of their means can be seen in Fig. 4(a) and Fig. 4(b) using MSP and teVG, respectively. The temporal dynamics of the two strongest sources at $170 \mathrm{~ms}$ are shown in Fig. 5. These correspond for both methods, to one source in the left and one in the right hemisphere. Fig. 5 shows in three panels the time courses of these two sources with their: a) $90 \%$ posterior distribution confidence intervals (only shown for MSP), and the resampling confidence intervals for the MSP (b) and teVG (c). Confidence intervals in figures 5(b) and (c) are estimated through 100 repetitions of split-half resampling of condition epochs, i.e. they arise from a total of $2 \cdot 100=200$ source reconstructions, see e.g., [23]. The differential responses found are close to zero in the pre-stimulus time window $([-200,0] \mathrm{ms})$ and peak at around $170 \mathrm{~ms}$ post-stimulus; the critical time scale for face perception [19]. The unbiased resampling based confidence intervals in the range 150-300 ms, are seen to be larger for MSP (5b) compared to teVG (5c). More specifically at $t=170 \mathrm{~ms}$ MSP has standard deviations of 0.42 (left dipole) and 0.47 (right dipole), where teVG has respectively 0.29 and 0.23 . We note that the posterior and the resampling based uncertainties show some similarity although quite different in 'statistical meaning' - the former quantifies the uncertainty within the model given the specific data set, while the latter represents the expected variability under repeated experiments.

The consistency of the locations of the recovered dipoles (the strongest left and right) found when using the whole data
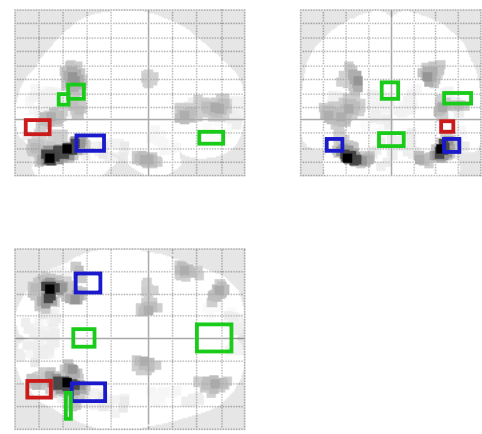

(a) MSP
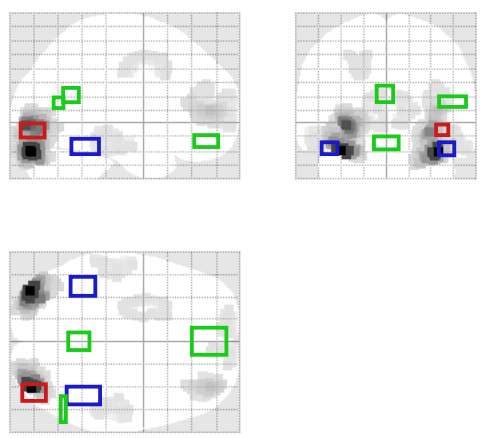

(b) teVG

Fig. 4. Glass brain view of source reconstruction of the differential response of faces and scrambled faces at $170 \mathrm{~ms}$ post-stimulus. 512 dipoles are shown for both methods. The activation found with fMRI of the subject's face specific response are illustrated with colored squares, see [21]. The blue are in the FFA, the red in the OFA, the remaining clusters discovered are shown in green.

set and in the split-halves is examined for the two methods. Compared to applying MSP to the mean difference across all epochs, the $2 \times 100$ splits recover on average the same left dipole $(29+43) / 2=36$ times and the right dipole $(12+15) / 2=13.5$ times. For teVG the locations of the splits' strongest dipoles comply $(58+57) / 2=57.5$ times for the left and $(69+65) / 2=67$ times for the right. Thus, in general teVG is more consistent with respect to the recovery of the strongest dipoles.

Inspecting in Fig. 4 the source locations for MSP and teVG, MSP seems to have a better overlap with the FFA (blue square in Fig. 4 as found by fMRI in [21]), whereas teVG seems to better locate the right occipital face area (red square). 


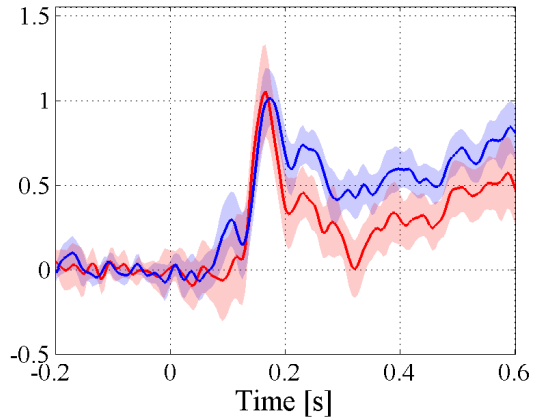

(a) MSP

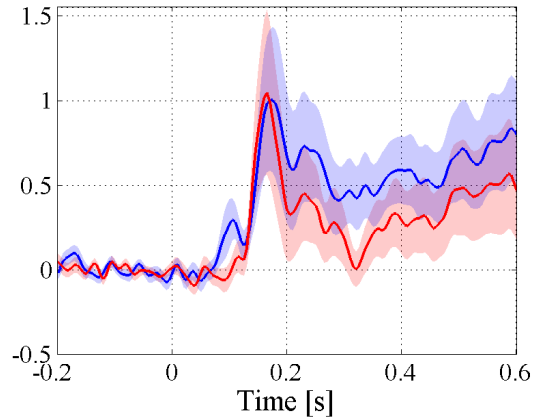

(b) MSP

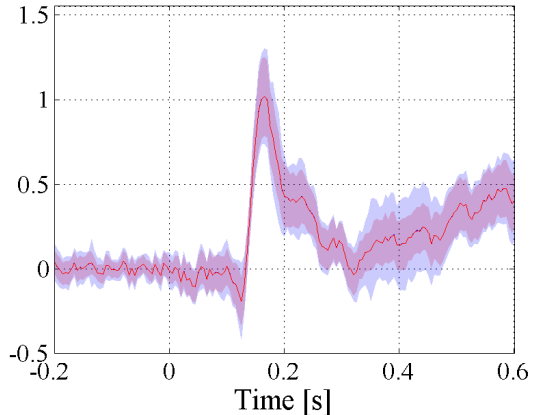

(c) teVG

Fig. 5. Source reconstruction of the face specific response. The two sources with strongest activity are shown, these are for both methods placed in the left (blue curve) and the right (red curve) hemisphere, respectively. The confidence interval seen in (a) are estimated from the posterior distribution, while they are in (b) and (c) calculated through 100 split-half resampling of the epochs. The magnitude of the sources have been scaled by the maximum value found in the source reconstruction on all epochs. Note that the activity of the two sources are very similar, especially for teVG where the two dipoles are exactly bilateral.

\section{CONClusion}

In conclusion we have shown that the MSP spatial basis function approach can be adapted easily to the Variational Garrote (VG). The VG applies cross-validation for estimation of its regularization strength, i.e, the effective sparsity level while the noise level is inferred, hence it does not require manual tuning of temporal complexity as in the MSP approach nor auxiliary signals for noise estimation as in Champagne. Finally, we used split-half resampling to provide an unbiased measure of the reproducibility of the activation time courses of the reconstructed spatial sources. For both MSP and teVG we found that the differential responses peaked around $170 \mathrm{~ms}$ post-stimulus, a timing that is well established for the face specific response [19]. The unbiased resampling based confidence intervals were found to be larger for MSP compared to teVG in the vicinity of this peak activity.

\section{ACKNOWLEDGMENT}

This work is supported by the Danish Lundbeck Foundation via the Center for Integrated Molecular Brain Imaging.

\section{REFERENCES}

[1] K. J. Friston, L. Harrison, J. Daunizeau, S. J. Kiebel, C. Phillips, N. Trujillo-Barreto, R. N. Henson, G. Flandin, and J. Mattout, "Multiple sparse priors for the M/EEG inverse problem." Neurolmage, vol. 39 , no. 3, pp. 1104-1120, 2008

[2] G. Strobbe, P. van Mierlo, M. De Vos, B. Mijović, H. Hallez, S. Van Huffel, J. D. López, and S. Vandenberghe, "Bayesian model selection of template forward models for EEG source reconstruction," NeuroImage, 2014.

[3] D. J. MacKay et al., "Bayesian nonlinear modeling for the prediction competition," ASHRAE transactions, vol. 100, no. 2, pp. 1053-1062, 1994.

[4] L. K. Hansen and C. E. Rasmussen, "Pruning from adaptive regularization," Neural Computation, vol. 6, no. 6, pp. 1223-1232, 1994.

[5] R. M. Neal, "Bayesian learning for neural networks," Ph.D. dissertation, University of Toronto, 1995

[6] D. P. Wipf, J. P. Owen, H. T. Attias, K. Sekihara, and S. S. Nagarajan, "Robust Bayesian estimation of the location, orientation, and time course of multiple correlated neural sources using MEG," NeuroImage, vol. 49, no. 1, pp. 641-655, 2010.

[7] J. P. Owen, D. P. Wipf, H. T. Attias, K. Sekihara, and S. S. Nagarajan, "Performance evaluation of the Champagne source reconstruction algorithm on simulated and real M/EEG data," Neuroimage, vol. 60, no. 1, pp. 305-323, 2012.

[8] M. Seeger and D. P. Wipf, "Variational Bayesian inference techniques," Signal Processing Magazine, IEEE, vol. 27, no. 6, pp. 81-91, 2010.
[9] D. P. Wipf and S. S. Nagarajan, "A new view of automatic relevance determination." in NIPS Advances in Neural Information Processing Systems 20, J. Platt, D. Koller, Y. Singer, and S. Roweis, Eds. New York: Curan Associates, 2007, pp. 1625-1632.

[10] R. D. Pascual-Marqui, C. M. Michel, and D. Lehmann, "Low resolution electromagnetic tomography: a new method for localizing electrical activity in the brain," International Journal of psychophysiology, vol. 18, no. 1, pp. 49-65, 1994.

[11] S. Baillet, J. C. Mosher, and R. M. Leahy, "Electromagnetic brain mapping," Signal Processing Magazine, IEEE, vol. 18, no. 6, pp. 14-30, 2001.

[12] H. Hallez, Vanrumste et al., "Review on solving the forward problem in EEG source analysis," Journal of neuroengineering and rehabilitation, vol. 4, no. 46, pp. 1-29, 2007.

[13] C. Stahlhut, M. Mørup, O. Winther, and L. K. Hansen, "Simultaneous EEG source and forward model reconstruction (sofomore) using a hierarchical bayesian approach," Journal of Signal Processing Systems, vol. 65, no. 3, pp. 431-444, 2011.

[14] A. Delorme, J. Palmer, J. Onton, R. Oostenveld, and S. Makeig, "Independent EEG sources are dipolar," PloS one, vol. 7, no. 2, p. e30135, 2012.

[15] H. J. Kappen and V. Gómez, "The Variational Garrote," Machine Learning, pp. 1-26, 2013.

[16] S. T. Hansen, C. Stahlhut, and L. K. Hansen, "Sparse Source EEG Imaging with the Variational Garrote," in Pattern Recognition in Neuroimaging (PRNI), 2013 International Workshop on. IEEE, 2013, pp. 106-109.

[17] _ _Expansion of the Variational Garrote to a Multiple Measurement Vectors Model," in Twelfth Scandinavian Conference on Artificial Intelligence. IOS, 2013, pp. 105-111.

[18] P. Nunez and R. Srinivasan, Electric fields of the brain: the neurophysics of EEG, 2nd ed. Oxford University Press, USA, 2006.

[19] R. N. Henson, Y. Goshen-Gottstein, T. Ganel, L. J. Otten, A. Quayle, and M. D. Rugg, "Electrophysiological and haemodynamic correlates of face perception, recognition and priming," Cerebral cortex, vol. 13, no. 7, pp. 793-805, 2003.

[20] P. A. Salin and J. Bullier, "Corticocortical connections in the visual system: structure and function." Physiological reviews, vol. 75 , no. 1, pp. 107-154, 1995.

[21] J. Ashburner, C.-C. Chen, R. Moran, R. N. Henson, V. Glauche, and C. Phillips, "SPM8 manual," The FIL Methods Group, Tech. Rep., 2012.

[22] A. Gramfort, T. Papadopoulo, E. Olivi, and M. Clerc, "Forward field computation with OpenMEEG," Computational intelligence and neuroscience, no. 1, pp. 1-13, 2011.

[23] S. Strother, S. La Conte, L. Kai Hansen, J. Anderson, J. Zhang, S. Pulapura, and D. Rottenberg, "Optimizing the fMRI data-processing pipeline using prediction and reproducibility performance metrics: I. A preliminary group analysis," Neuroimage, vol. 23, pp. S196-S207, 2004. 